\title{
Nonmagnetic impurities in skyrmion racetrack memory
}

\author{
M. N. Potkina ${ }^{1,2,3}$, I. S. Lobanov ${ }^{1,2}$, V. M. Uzdin ${ }^{1,2}$ \\ ${ }^{1}$ ITMO University, Kronverkskiy, 49, St. Petersburg, 197101, Russia \\ ${ }^{2}$ St. Petersburg State University, St. Petersburg, 198504, Russia \\ ${ }^{3}$ Science Institute and Faculty of Physical Sciences, Univ. of Iceland, 107 Reykjavík, Iceland \\ potkina.maria@yandex.ru, lobanov.igor@gmail.com, v_uzdin@mail.ru
}

PACS 75.10.Hk, 75.75.-c, 82.20.Pm

DOI 10.17586/2220-8054-2020-11-6-628-635

\begin{abstract}
The influence of non-magnetic defects of different sizes on the stability and anchoring of skyrmions in race track memory devices has been investigated. The energy surface of the system was built on the basis of the generalized Heisenberg model, which includes exchange, DzyaloshinskiiMoriya interaction, anisotropy, and an external magnetic field. Minima and saddle points on the energy surface are used to estimate quantitatively the stability and pinning effects for skyrmions. The activation energies for attachment and detachment of skyrmions from defects, collapse and nucleation of skyrmions on a nonmagnetic impurity on a track of finite width are calculated. The joint effect of defects and the proximity of sample boundaries on the stability and localization of skyrmions has been studied. It is shown that skyrmion race track memory can only work if the track width is much greater than four times the skyrmion radius, and the spatial size of defects that can pin a skyrmion is small compared to its own size. Otherwise, the skyrmion will annihilate instead of moving under the action of the spin-polarized current.
\end{abstract}

Keywords: skyrmion, racetrack memory, impurity, pinning, transition state, stability.

Received: 1 October 2020

Revised: 27 October 2020

\section{Introduction}

Domain wall racetrack memory (DW-RM) was proposed in 2008 [1] as an alternative to hard disk drive (HDD) storage devices to prevent the use of slow mechanical parts and replace them with the movement of domain walls in ferromagnetic nanowires. Since then, hard drives are being supplanted by solid state drives (SSDs) which store data in semiconductor cells, that contain no moving parts and have a read/write speed much faster than HDD. DW-RM is still under development. Many new designs have been proposed [2], which in particular promises higher storage densities than SSDs, due to the three-dimensional organization of magnetic shift registers. After experimental observations of magnetic skyrmions (Sks), they were proposed as a better alternative to domain walls (DWs) as a storage unit for the magnetic racetrack memory [3]. Sks are chiral excitation-like particles ranging in size from a few nanometers to micrometers [4] and are stable up to room temperature [5]. Sk's small size makes them promising for data storage applications [6,7]. The first studies showed that the pinning by impurities for Sk is very low in contrast to the case of DW [8]. Therefore, critical current density in Sk based racetrack memory (Sk-RM) for performing the shift in the register can be much smaller than that of the DW-RM, and overall power consumption can be reduced. However, theoretical calculations have shown that Sk can be captured by a non-magnetic impurity if the current density is sufficiently small [9]. At present, the problem of the interaction of Sk with impurities is considered very important in the context of the Sk-RM construction [10,11].

Sk-RM memory control consists of several steps [12]. Each of them was implemented using at least one scheme: writing [13], shift operation [14], and reading [15]. In order to design a reliable device, constrained geometry and spatial defects should be taken into account from the very beginning. Thermal instability and Sk escape through the boundary were considered in [16], demonstrating that for certain parameters Sk in the track lives hours and more. Another important issue is the presence of impurities and point defects, which is usually considered an inevitable disadvantage. A controllable pinning however can be a functional part of the Sk-RM design [17,18].

Spin transfer torque (STT) is commonly used to move skyrmions in the Sk-RT memory. Although there is usually a certain angle between the direction of the current flow and the velocity of the Sk, called the Hall angle, for certain parameters, when the Hilbert damping is equal to the non-adiabatic STT parameter, the Sk velocity can be parallel to the current [7]. For larger current density and multi-Sk states, more complex behavior such as the rotation of the Sk lattices [19] may appear. Zero Hall angle can be achieved with antiferromagnetic Sk [11,20] or antiskyrmions for a specific direction of current flow [21,22].

It is shown that various types of point defects have a different effect on Sk under the current flow. Local disturbance of the anisotropy has little effect on the motion of Sk, since Sk moves around the impurities, avoiding their 
capture [8]. However, in disordered magnets with grain defects, new phases, such as Sk multiplication/annihilation and Sk segregation, appear together with the localized and depinned Sk [23] phases. Nonmagnetic impurities can capture Sk at low currents, but the pinning effect strongly depends on the parameters of the system. With certain parameters, even infinitesimal currents can separate the skyrmion from the non-magnetic impurity [9].

Wide range of point impurities demonstrating anti- and ferromagnetic coupling with the host material was analyzed in [24] on the basis of ab initio calculations. Different local magnetization and other magnetic characteristic make the impurity repulsive or attractive with respect to Sk, can give different stable positions within Sk, result in different dynamics [25]. Several ways to use impurities in Sk-RM have been proposed. The Sk velocity can unexpectedly be increased in the presence of non-magnetic impurity [9] or a linear defect [26]. The impurities can be used to make repelling and attractive rails for Sk guides [24,27]. Simultaneous interaction of Sk with several impurities not only change its velocity, but also may lead to new qualitative behaviour [23]. That means that the effect of the restrains should be taken into account especially in restricted geometries such as Sk tracks. Recent modifications of the SkRM with enhanced capabilities have more complex geometry than the original design, hence, the effect of geometric frustration can be strongly pronounced in the devices, such as two-lane Sk-RM [28], or random access Sk-RM [29].

In this work, we study the interaction of Sk with an impurity inside a track of different widths. We show that the track width significantly affects the Sk stability and the activation energies of pinning and depinning. The pinned Sks in narrow tracks are not only less stable, but also have a greater barrier for dissociation. Since the critical current required to overcome the activation barrier should have the same qualitative behavior [9], we have concluded that most energy efficient Sk-RM devices should have a track width significantly greater than two Sk diameters. We also study the effect of the size of an atomic-resolved non-magnetic impurity on the activation energies of annihilation/nucleation, pinning/depinning processes. Sk remains stable even for impurities with a size of half the Sk radius. Both nucleation and annihilation barriers decrease with an increase in the impurity size, which makes the formation of Sk on impurities more probable, but the Sk decay rate also increases. The Sk attachment and detachment barrier increases with the impurity size, making the pinning more strong. Since anchoring Sk in the read and write phase may be desirable, this can be achieved by placing a sufficiently large non-magnetic defect at the appropriate location in the track. Both increase of the impurity size and miniaturization of the track (for constant Sk size) lead to rapid growth of depinning barrier and energy consumption by Sk-RM devices.

\section{Method and simulated system}

A thin magnetic film is modelled within the generalized Heisenberg model, which is a standard tool for Sk simulation with atomistic resolution. Exchange interaction, Dzyaloshinskii-Moriya interaction, anisotropy energy and Zeeman energy of interaction with an external field $\mathbf{B}$ are included in the total energy $E$ :

$$
E=-J \sum_{\langle i, j\rangle} \mathbf{S}_{i} \cdot \mathbf{S}_{j}-\sum_{\langle i, j\rangle} \mathbf{D}_{i j} \cdot\left(\mathbf{S}_{i} \times \mathbf{S}_{j}\right)-\mu \sum_{i} \mathbf{B} \cdot \mathbf{S}_{i}-K \sum_{i} S_{i, z}^{2},
$$

where summation is taken over all pairs $\langle i, j\rangle$ of nearest neighbours atoms, and over all atoms $i$ of the triangular lattice except for impurities. Here, $J$ is the exchange parameter, $\mathbf{D}_{i j}$ is the Dzyaloshinskii-Moriya vector lying in the film plane perpendicular to the vector connecting atomic sites $i$ and $j, K>0$ is the anisotropy constant for easy axis $z$ perpendicular to the film, $\mathbf{B}$ is the external magnetic field parallel to the $\mathbf{z}$-axis and $\mathbf{S}_{i}$ is a three-dimensional vector of unit length in the direction of the magnetic moment at the site $i$. The magnitude of the moment, $\mu$, is assumed to be the same for all sites; impurities are considered as holes without magnetic moments in the lattice. The numerical values of the parameters correspond to experimentally observed skyrmions in the $\mathrm{Pd} / \mathrm{Fe} / \mathrm{Ir}(111)$ system: $\mu B=0.093 J, K=0.07 J, D=\left|\mathbf{D}_{i j}\right|=0.32 J, J=7 \mathrm{meV}$ [30]. The parameters correspond to isolated free Sk of radius $4 a=1.08 \mathrm{~nm}$, where $a=0.27 \mathrm{~nm}$ is the lattice constant. To simulate a track of finite width we apply periodic boundary conditions along the x-axis and assume free boundaries along the y-axis. Track length $l_{x}=60 a$ is the same for all simulations and is large enough to avoid Sk self interaction through the boundary. Track width $l_{y}=W a \sin \frac{\pi}{3}$ is a system parameter and varies from $W=19$ to $W=61$, where the smallest width corresponds to the smallest stable Sk in the track, and for the largest $W$ free boundary effect on the Sk energy is less than $0.007 \%$. Nonmagnetic impurities are simulated by removing some of the spins forming a single cluster.

(Meta)stable states correspond to local minima on the multidimensional energy surface defined by the equation (1) for $E$ as a function of the quantities determining the direction of all magnetic moments $\mathbf{S}$. For the given set of parameters the ground state corresponds to the ferromagnetic (FM) state with a perturbation near the impurity and rotating moments on the free boundary. Sk on an impurity and free Sk form two metastable states, where the preferred position of the impurity inside Sk is where the magnetic moments lie in the plane of the film. Sk shape is affected by the impurity. Metastable states were computed using conjugated gradient method in Cartesian coordinates [31]. Activation barriers for transition between pinned, depinned Sk and FM phase were computed using minimum energy 
path (MEP), which gives the most probable transition scenario [32]. The maximum along the MEP determines the first-order saddle point on the energy surface and the activation barriers between states. A variant of geodesic nudged elastic band method [33] in Cartesian coordinates was applied to compute MEP with gradient at stationary points smaller than $10^{-6} \mathrm{~J} / \mathrm{rad}$.

\section{Effects of impurity size and track width}

We consider a non-magnetic impurity consisting of 1, 3, 4, 7 and 8 non-magnetic atoms or vacancies that form a compact group on a two-dimensional triangular lattice. According to the previous studies [9], stable positions of the impurity on the pinned Sk are given by the minima of the pinning potential, which roughly corresponds to the local maxima of energy density of the free Sk, neglecting change of the Sk shape under pinning. The Sk on the cluster of impurities tends to rotate and shift to maximize the sum of energy contributions of all spins in Sk replaced by the vacancies. Fig. 1 shows the relative position of the Sk and the impurity corresponding to the minimum of energy. For all considered sizes and shapes of the defect, the impurity cluster is located near the region, where the magnetization vector lies in the film plane. For the elongated clusters the long axis tends to be along curve of constant energy density. Such arrangement was observed experimentally by scanning tunneling microscopy for skyrmions in a PdFe bilayer on the Ir (111) surface [34]. The energy of a Sk at an impurity, as our calculation shows, is lower than that far from the defect, which may be the possible explanation, why Sks are often created on the impurities.

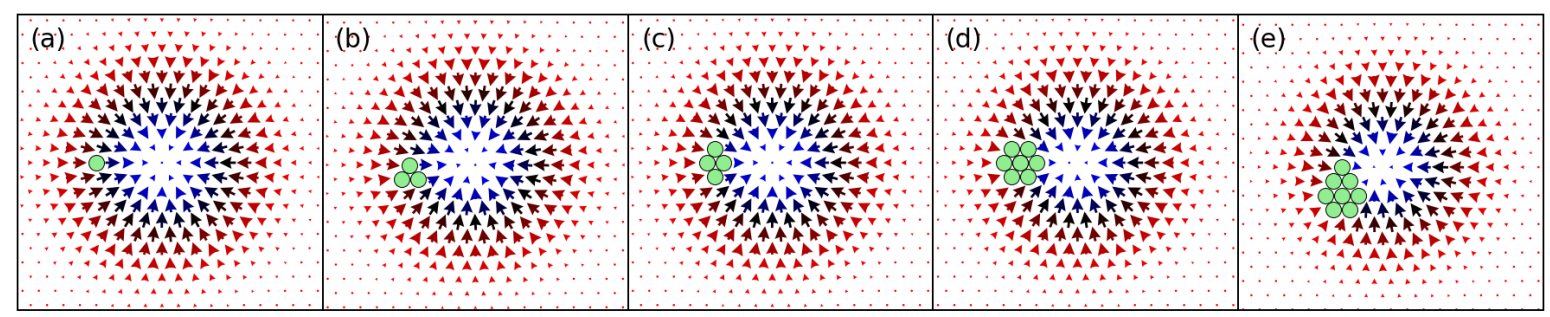

FIG. 1. Location of a skyrmion at non-magnetic defects consisting of (a) 1, (b) 3, (c) 4, (d) 7, (e) 8 atoms, corresponding to the minimum energy. Arrows show in-plane direction of magnetic moments, color denotes their out of plane component (red is for moment oriented along the external field, and blue for the opposite orientation). Impurities are marked by green dots.

Previous research has shown that repulsion from the free boundary significantly affects the shape of Sk and its stability up to disappearance of Sk metastable state for very narrow tracks [35, 36]. Despite the fact that Sk on the impurity has lower energy than the free Sk, the activation barrier for collapse of the pinned Sk is also lower than for the free one, making the pinned Sk less stable. In the proposed circuits [24], where Sk motion is directed by an impurities chain, as well as in Sk-RM [3], both interaction with impurities and effect of the boundary simultaneously affect Sk stability. The question arises how impurities in combination with track boundaries will influence the skyrmion localization and collapse. To answer this question, we calculated the MEP and energy barriers for pinning/depinning and for annihilation/nucleation processes for each impurity size shown in Fig.1. The MEPs for attachment of skyrmion to seven-atomic impurity (1-2-3) and subsequent collapse (3-4-5) are shown in Fig. 2. Magnetic configurations along the MEPs are shown in insets. The pinned Sk state (3) has lower energy than the free Sk (1) in all considered cases, which in turn is significantly larger than the energy of the FM state meaning that the Sk phase corresponds to isolated Sks. Energy of the transition state (TS) (2) for the pinning process depends on the defect size and is higher for larger defects and narrower tracks. The energy of TS (4) for the annihilation process increases with the size of the impurity as well as with the compression of the track. Relative energies of TS (2) and TS (4) depend on the system parameters as will be shown below. Sk escape through the boundary is also possible, however the process typically proceeds in two steps: the depinning and after that the escape, except of very narrow tracks. Escape of the free Sk through the boundary was studied in [36], where crossover between collapse and escape is found depending on the external field.

The barriers for localization of a skyrmion on a defect (a) and for detachment from it (b) are shown in Fig. 3 for various impurity sizes as a function of track width $W$. For any particular size of the impurity the pinning barrier is less than barrier for the detachment, which is in agreement with the previous results $[20,36]$. The difference in the activation energies means that Sk will occupy nonmagnetic impurities if thermal energy will be enough to overcome the barrier for attachment but not so large to dissociate from defect immediately.

Both barriers for the pinning of Sk to the defect and for the reverse process increase with the defect size. Therefore, pinning and depinning are less likely for larger defects. The barrier for the pinning is practically independent of the 


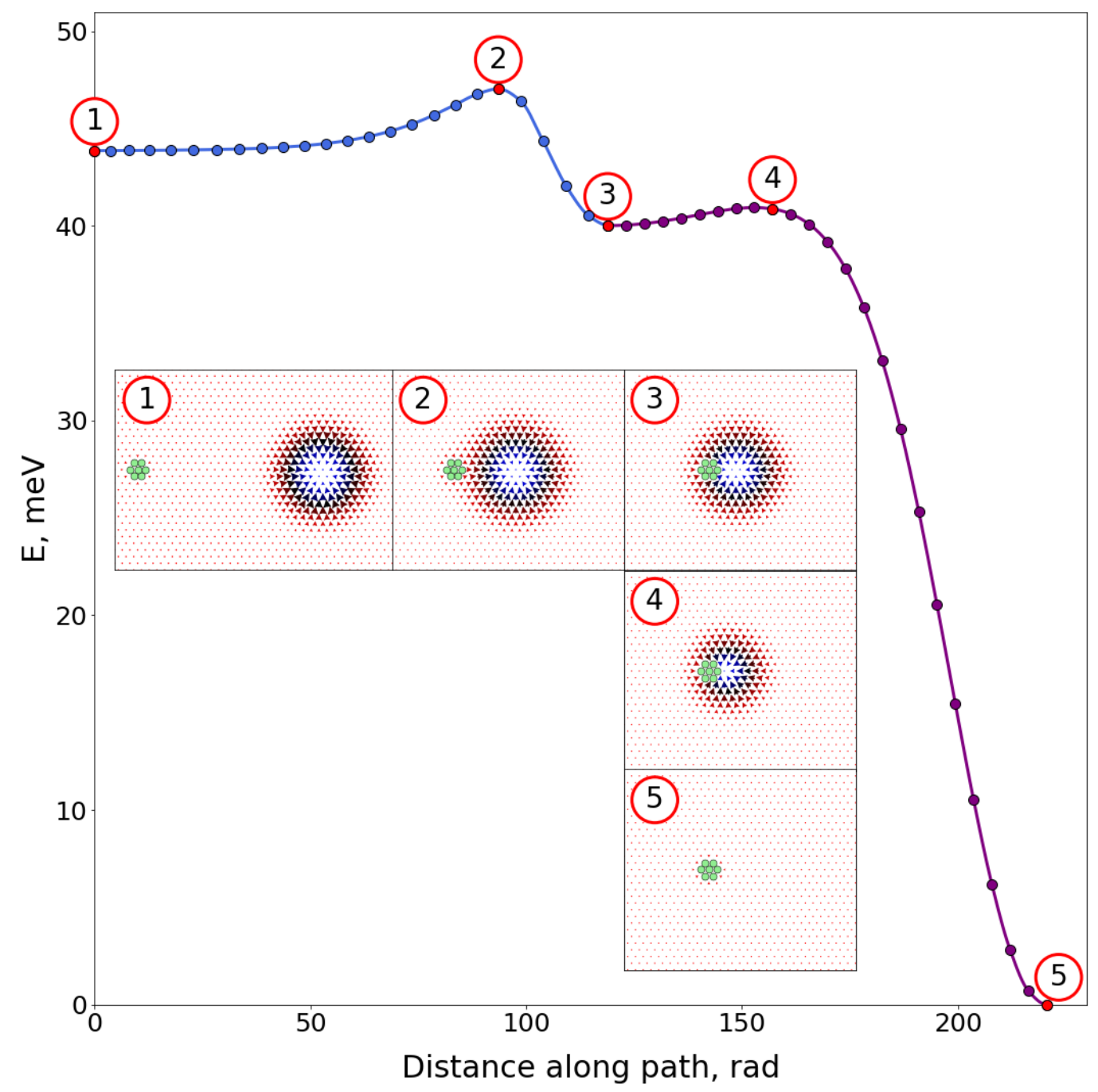

FIG. 2. Curve 1-2-3 represents the MEP between a skyrmion far from the defect and a skyrmion localized at a 7-atom nonmagnetic defect. Curve 3-4-5 represents the MEP between the skyrmion localized at the defect and the FM state with impurity. The insets show magnetic configurations along the MEPs. States 2 and 4 correspond to saddle points. Skyrmion is localized inside a track with a width of 61 atoms.

width of the track, being slightly smaller for narrow tracks. On the other hand, the activation energy for detachment rapidly increases when the track width becomes less than five Sk diameters, sharply enlarging the strength of fixation of the defect in the narrow track. The effect manifests itself for nonmagnetic defects of all sizes, although it is less pronounced for large defects. Since the critical current for the Sk motion over the nonmagnetic impurities can be estimated by the depinning barrier, the overall energy consumption by Sk-RM will be significantly higher in narrow tracks than for free Sks.

As track width is decreased, Sk decay rate became larger. When both the proximity of the sample boundary and non-magnetic defects act on Sk, its stability is expected to decrease even more. The smallest track width at which a Sk may exist increases with increase of the defect size, e.g. Sk localized at eight-atomic defect is not stable for $W<50$, but Sk on the single site defect is stable up to $W=20$. The non-magnetic defect significantly reduces the activation barrier for the collapse of a skyrmion in comparison with annihilation in a homogeneous medium. For example, the barrier for the decay of a skyrmion on a defect of seven atoms is less than $1 \mathrm{meV}$; without such a defect on a track of the same width, the barrier is $42 \mathrm{meV}[35,36]$. The energy barrier for the collapse decreases with track width for each impurity size, as shown in Fig. 4a. The decrease is faster for smaller defects and becomes fast when the track diameter 

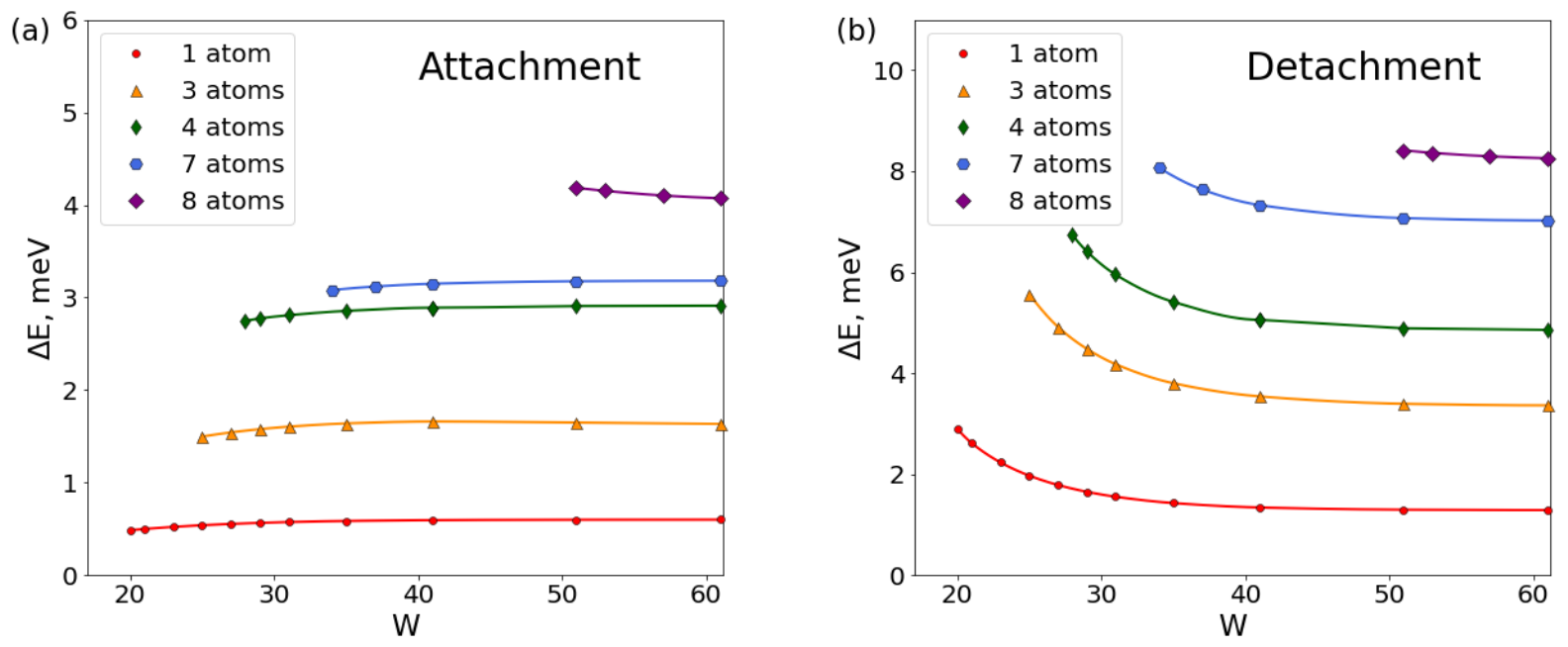

FIG. 3. Energy barrier for attachment (a) and detachment (b) of skyrmion from a defect of different sizes, depending on the width of the track W.
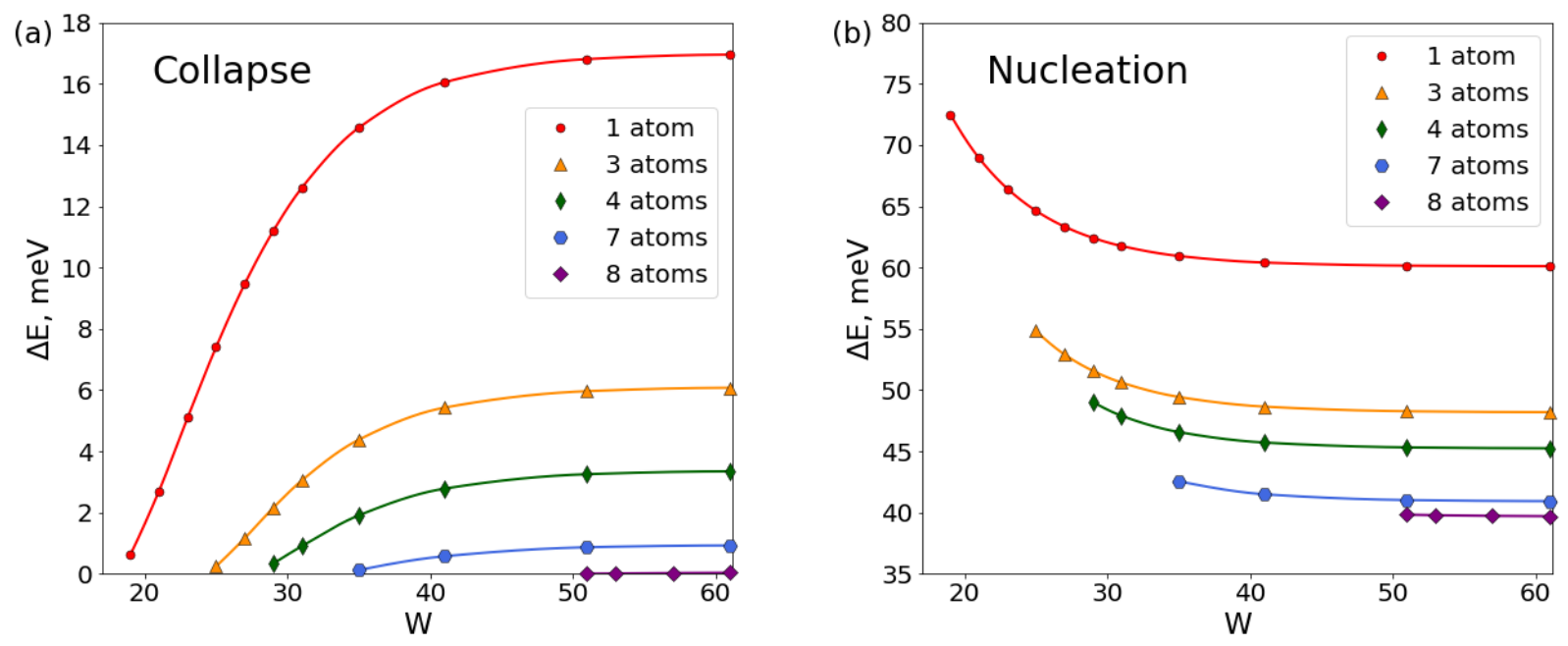

FIG. 4. Energy barrier for annihilation (a) and nucleation (b) of a skyrmion on impurities of different sizes depending on the track width $\mathrm{W}$.

is less than five Sk for all defect sizes. This means that in narrow lanes, Sk is not only difficult to separate from the defect, but Sk is likely to be destroyed during separation.

The nucleation barrier decreases with the size of the defect in the same way as the annihilation barrier. Therefore, the state near the large defect is regularly perturbed creating new Sks and annihilating them. In the presence of a depinning current, such impurity can be used as Sk generators. In contrast to the behavior of the activation energy for the collapse, the nucleation barrier increases with decreasing track width (Fig. 4b); therefore, the Sk concentration in narrow tracks will be extremely low. The influence of boundaries is less for localization-delocalization processes than for collapse-nucleation ones, because the configuration of the lateral part of a skyrmion interacting with an impurity changes weakly when a skyrmion is compressed by boundaries, while the entire skyrmion becomes elliptical and has a smaller radius.

A pinned Sk can be removed from the impurity by current flow or it can come off due to thermal vibrations, but it can also collapse during the process. In Sk-RM devices Sk collapse is undesirable, except of the read/write stages, 

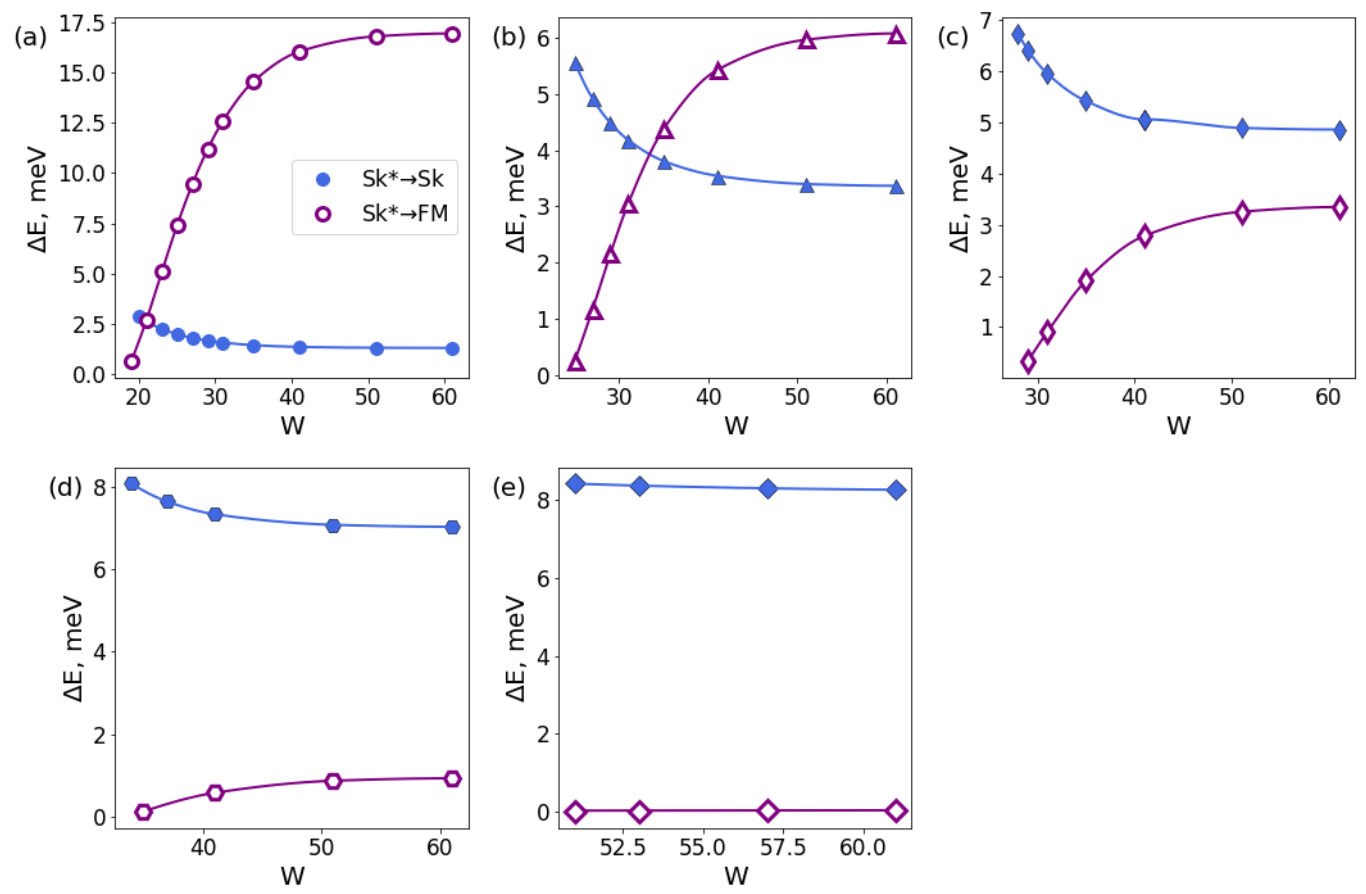

FIG. 5. Dependence of energy barriers for the detachment of a skyrmion from a defect and for annihilation of a skyrmion for various impurity sizes: (a) 1 atom, (b) 3 atoms, (c) 4 atoms, (d) 7 atoms, (e) 8 atoms on the width of the track.
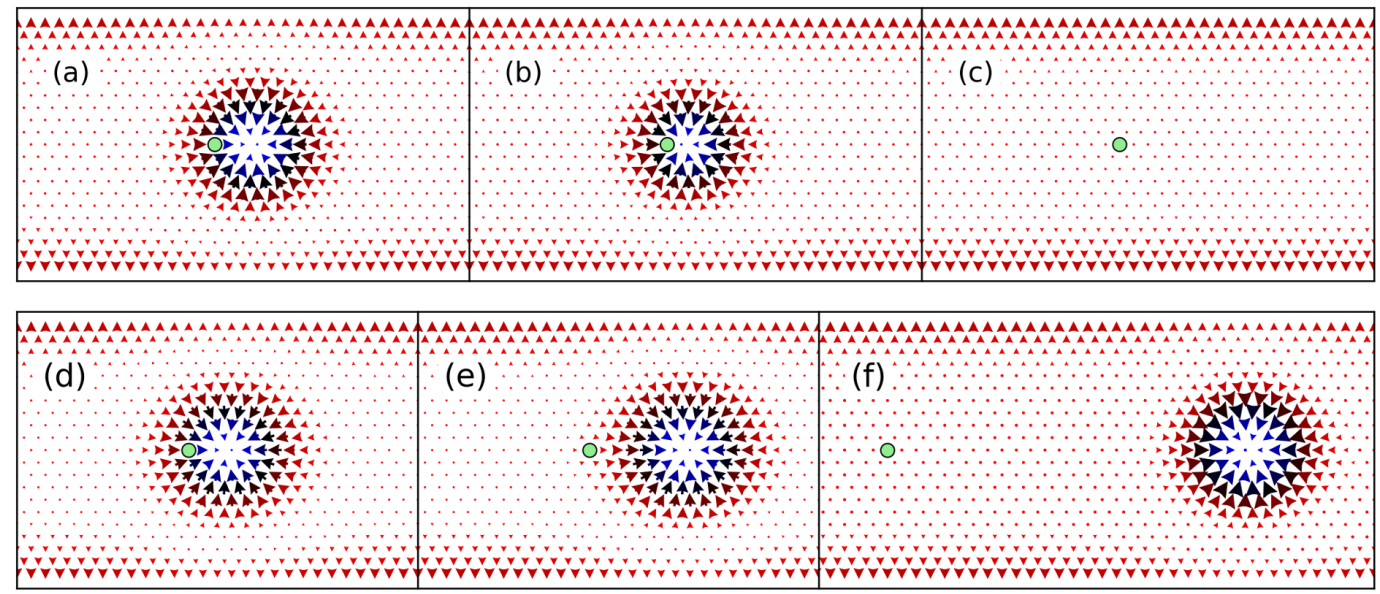

FIG. 6. Upper row: Configurations along MEP for skyrmion annihilation at one-atomic impurity: (a) initial minimum corresponding to skyrmion localized at impurity (b) saddle point (c) final minimum corresponding to FM state with impurity. Lower row: Configurations along MEP for skyrmion detachment from one-atomic impurity: (d) initial minimum corresponding to skyrmion localized at impurity (e) saddle point (f) final minimum corresponding to skyrmion far from impurity.

hence the depinning barrier is expected to be lower than the collapse activation energy. The actual barriers relation is however parameters dependent. The barriers for the Sk detachment and for the collapse at the impurity are compared in Fig. 5 as a function of the track width. For small defects (consisting of one and three atoms), the track width determines which process will be more probable: with a large width, the skyrmion is more likely to detach from impurity, and with a small one, it more likely collapses. There is crossover between these processes, the corresponding track width is the smallest one applicable in Sk-RM devices. For example, for one-atomic impurity the crossover occurs at the track width corresponding to $W=21$. For this situation Fig. 6 shows the magnetic configurations along the MEPs for 
collapse and for detachment from a defect: the initial state, the saddle point, and the final state. But, as shown in Fig. 5 $\mathrm{c}, \mathrm{d}, \mathrm{e}$, if the defect is large, the barrier for collapse is always smaller than for detachment, and under random influences, for example, due to thermal fluctuations, skyrmions will likely collapse without dissociation from the defect. Using the activation barrier as a loose estimate for the critical current, we conjecture that Sk-RM is not functioning in the presence of defects larger than width of Sk domain wall, approximately.

\section{Acknowledgements}

This work was funded by Russian Science Foundation (Grant 19-42-06302). MNP acknowledges the Icelandic Research Fund for financial support.

\section{References}

[1] Parkin S.S.P., Hayashi M., Thomas L. Magnetic domain-wall racetrack memory. Science, 2008, 320(5873), P. 190-194.

[2] Mittal S. A survey of techniques for architecting processor components using domain-wall memory. ACM Journal on Emerging Technologies in Computing Systems (JETC), 2016, 13(2), P. 1-25.

[3] Fert A., Cros V., Sampaio J. Skyrmions on the track. Nature Nanotechnology, 2013, 8, P. 152-156.

[4] Wiesendanger R. Nanoscale magnetic skyrmions in metallic films and multilayers: a new twist for spintronics. Nature Reviews Materials, 2016, 1(7), P. 16044.

[5] Soumyanarayanan A., Raju M., Oyarce A.L.G., Tan A.K.C., Im M.Y., Petrović A.P., Ho P., Khoo K.H., Tran M., Gan C.K., Ernult F., Panagopoulos C. Tunable room-temperature magnetic skyrmions in Ir/Fe/Co/Pt multilayers. Nature Materials, 2017, 16(9) P. 898-904.

[6] Fert A., Reyren N., Cros V. Magnetic skyrmions: advances in physics and potential applications. Nature Reviews Materials, 2017,2 , P. 17031.

[7] Finocchio G., Büttner F., Tomasello R., Carpentieri M., Kläui M. Magnetic skyrmions: from fundamental to applications. Journal of Physics D: Applied Physics, 2016, 49(42), P. 423001.

[8] Iwasaki J., Mochizuki M., Nagaosa N. Universal current-velocity relation of skyrmion motion in chiral magnets. Nature Communications, 2013, 4(1), P. 1463

[9] Müller J., Rosch A. Capturing of a magnetic skyrmion with a hole. Physical Review B, 2015, 91(5), P. 054410.

[10] Back C.H., Cros V., Ebert H., Everschor-Sitte K., Fert A., Garst M., Ma T., Mankovsky S., Monchesky T., Mostovoy M.V., Nagaosa N. The 2020 skyrmionics roadmap. Journal of Physics D: Applied Physics, 2020, 53(36), P. 363001.

[11] Everschor-Sitte K., Masell J., Reeve R.M., Kläui M. Perspective: Magnetic skyrmions - Overview of recent progress in an active research field. Journal of Applied Physics, 2018, 124(24), P. 240901.

[12] Sampaio J., Cros V., Rohart S., Thiaville A., Fert A. Nucleation, stability and current-induced motion of isolated magnetic skyrmions in nanostructures. Nature Nanotechnology, 2013, 8(11), P. 839.

[13] Romming N., Hanneken C., Menzel M., Bickel J.E., Wolter B., von Bergmann K., Kubetzka A., Wiesendanger R. Writing and Deleting Single Magnetic Skyrmions. Science, 2013, 341(6146), P. 636-639.

[14] Hayashi M., Thomas L., Moriya R., Rettner Ch., Parkin S.S.P. Current-Controlled Magnetic Domain-Wall Nanowire Shift Register. Science, 2008, 320(5873), P. 209-211.

[15] Crum D.M., Bouhassoune M., Bouaziz J., Schweflinghaus B., Blügel S., Lounis S. Perpendicular reading of single confined magnetic skyrmions. Nature Communications, 2015, 6(1), P. 8541.

[16] Bessarab P.F., Müller G.P., Lobanov I.S., Rybakov F.N., Kiselev N.S., Jónsson H., Uzdin V.M., Blügel S., Bergqvist L., Delin A. Lifetime of racetrack skyrmions. Scientific Reports, 2018, 8(1), P. 3433.

[17] Yan Z.R., Liu Y.Z., Guang Y., Feng J.F., Lake R.K., Yu G.Q., Han X.F. Robust Skyrmion Shift Device Through Engineering the Local Exchange-Bias Field. Physical Review Applied, 2020, 14(4), P. 044008.

[18] Suess D., Vogler C., Bruckner F., Heistracher P., Slanovc F., Abert C. Spin Torque Efficiency and Analytic Error Rate Estimates of Skyrmion Racetrack Memory. Scientific Reports, 2019, 9(1), P. 4827.

[19] Everschor K., Garst M., Binz B., Jonietz F., Mühlbauer S., Pfleiderer C., Rosch A. Rotating skyrmion lattices by spin torques and field or temperature gradients. Physical Review B, 2012, 86(5), P. 054432.

[20] Potkina M.N., Lobanov I.S., Jnsson H., Uzdin V.M. Skyrmions in antiferromagnets: Thermal stability and the effect of external field and impurities. Journal of Applied Physics, 2020, 127(21), P. 213906.

[21] Potkina M.N., Lobanov I.S., Tretiakov O.A, Jónsson H, Uzdin V.M. Antiskyrmions in Ferromagnets and Antiferromagnets: Stability and Dynamics, 2019. arXiv preprint, arXiv:1906.06383v2.

[22] Potkina M.N., Lobanov I.S., Tretiakov O.A., Jnsson H., Uzdin V.M. Stability of Long-lived Antiskyrmions in Mn-Pt-Sn Material. Physical Review B, 2020, 102(13), P. 134430.

[23] Koshibae W., Nagaosa N. Theory of current-driven skyrmions in disordered magnets. Scientific Reports, $2018,8(1)$, P. 6328.

[24] Fernandes I.L., Bouaziz J., Blügel S., Lounis S. Universality of defect-skyrmion interaction profiles. Nature Communications, 2018, 9(1), P. 4395.

[25] Fernandes I.L, Chico J., Lounis S. Impurity-dependent gyrotropic motion, deflection and pinning of current-driven ultrasmall skyrmions in $\mathrm{PdFe} / \mathrm{Ir}(111)$ surface. Journal of Physics: Condensed Matter, 2020, 32(42), P. 425802.

[26] Castell-Queralt J., González-Gómez L., Del-Valle N., Sanchez A., Navau C. Accelerating, guiding, and compressing skyrmions by defect rails. Nanoscale, 2019, 11(26), P. 12589-12594.

[27] Stosic D., Ludermir B.T., Miloevi V.M. Pinning of magnetic skyrmions in a monolayer Co film on Pt(111): Theoretical characterization and exemplified utilization. Physical Review B, 2017, 96(21), P. 214403.

[28] Müller J. Magnetic skyrmions on a two-lane racetrack. New Journal of Physics, 2017, 19, P. 1740006.

[29] Zhu D., Kang W., Li S., Huang Y., Zhang X., Zhou Y., Zhao W. Skyrmion Racetrack Memory With Random Information Update/Deletion/Insertion. IEEE Transactions on Electron Devices, 2018, 65(1), P. 87-95. 
[30] Hagemeister J., Romming N., von Bergmann K., Vedmedenko E.Y., Wiesendanger R. Stability of single skyrmionic bits. Nature Communications, 2015, 6(1), P. 8455.

[31] Lobanov I.S., Uzdin V.M. The lifetime of big size topological chiral magnetic states. Estimation of the pre-exponential factor in the Arrhenius law, 2020. arXiv preprint, arXiv:2008.06754 [cond-mat.mtrl-sci]

[32] Bessarab P.F., Uzdin V.M., Jónsson H. Harmonic transition-state theory of thermal spin transitions. Physical Review B, 2012, 85(18), P. 184409.

[33] Bessarab P.F., Uzdin V.M., Jnsson H. Method for finding mechanism and activation energy of magnetic transitions, applied to skyrmion and antivortex annihilation. Computer Physics Communications, 2015, 196, P. 335-347.

[34] Hanneken C., Kubetzka A., von Bergmann K., Wiesendanger R. Pinning and movement of individual nanoscale magnetic skyrmions via defects. New Journal of Physics, 2016, 18(5), P. 055009.

[35] Uzdin V.M., Potkina M.N., Lobanov I.S., Bessarab P.F., Jónsson H. The effect of confinement and defects on the thermal stability of skyrmions. Physica B: Condensed Matter, 2018, 549, P. 6-9.

[36] Uzdin V.M., Potkina M.N., Lobanov I.S., Bessarab P.F., Jónsson H. Energy surface and lifetime of magnetic skyrmions. Journal of Magnetism and Magnetic Materials, 2018, 459, P. 236-240. 